Article

\title{
A Combination of TsHARP and Thin Plate Spline Interpolation for Spatial Sharpening of Thermal Imagery
}

\section{Xuehong Chen ${ }^{1}$, Wentao Li ${ }^{1}$, Jin Chen ${ }^{1}{ }^{*}$, Yuhan Rao ${ }^{1}$ and Yasushi Yamaguchi ${ }^{2}$}

1 State Key Laboratory of Earth Surface Processes and Resource Ecology,

Beijing Normal University, Beijing 100875, China; E-Mails: chenxuehong@bnu.edu.cn (X.C.); 200911131027@mail.bnu.edu.cn (W.L.); yuhan.rao@gmail.com (Y.R.)

2 Graduate School of Environmental Studies, Nagoya University, Nagoya 464-8601, Japan;

E-Mail: yasushi@nagoya-u.jp

* Author to whom correspondence should be addressed; E-Mail: chenjin@ @nu.edu.cn;

Tel.: +86-135-2288-9711.

Received: 25 November 2013; in revised form: 20 February 2014 / Accepted: 6 March 2014 /

Published: 28 March 2014

\begin{abstract}
There have been many studies and much attention paid to spatial sharpening for thermal imagery. Among them, TsHARP, based on the good correlation between vegetation index and land surface temperature (LST), is regarded as a standard technique because of its operational simplicity and effectiveness. However, as LST is affected by other factors (e.g., soil moisture) in the areas with low vegetation cover, these areas cannot be well sharpened by TsHARP. Thin plate spline (TPS) is another popular downscaling technique for surface data. It has been shown to be accurate and robust for different datasets; however, it has not yet been attempted in thermal sharpening. This paper proposes to combine the TsHARP and TPS methods to enhance the advantages of each. The spatially explicit errors of these two methods were firstly estimated in theory, and then the results of TPS and TsHARP were combined with the estimation of their errors. The experiments performed across various landscapes and data showed that the proposed combined method performs more robustly and accurately than TsHARP.
\end{abstract}

Keywords: thermal sharpening; land surface temperature; TsHARP; thin plate spline; error estimation 


\section{Introduction}

Land surface temperature (LST) is an indispensable parameter for radiation budget estimation, drought monitoring, urban heat island assessment and other global change related studies [1-4]. Due to the technical limitations of instrument design, the spatial resolution of the thermal infrared (TIR) band is unfortunately coarser than that of the visible-near infrared (VNIR) bands for most remote sensing platforms [5]. As LST data are required at a relatively finer resolution for many applications, such as urban heat island analysis, precise agriculture monitoring, etc. [5,6], considerable efforts have been devoted to sharpen the thermal imagery using VNIR bands [7,8]. Among them, the TsHARP method $[9,10]$ is the most popular because of its operational simplicity and effectiveness [11]. The effectiveness of TsHARP is based on the fact that vegetation cover, mainly represented by Normalized Difference Vegetation Index (NDVI), is the most important factor for determining the LST of many landscapes. Thus, this method assumes that the relationship between LST and NDVI is unique across a given imaging scene and independent at different scales. Therefore, the NDVI-LST relationship that has been established at thermal image can be applied to VNIR image, by which the LST image can be sharpened to the higher VNIR resolution. The effectiveness of TsHARP has been demonstrated in previous studies $[12,13]$.

However, recent studies also reported some disadvantages of TsHARP. The scale effect of the NDVI-LST relationship cannot always be disregarded, which could lead to a sizable error for areas dominated by natural vegetation [14]. Moreover, NDVI is not the unique parameter that determines LST. A triangular scatterplot in NDVI-LST feature space does commonly exist in many images $[15,16]$. It implies that LST varies largely by other factors (e.g., soil moisture, albedo) in areas with low NDVI values. Although this issue can be addressed to some extent by residual correction, it is still a significant source of errors for TsHARP in heterogeneous areas [13]. Therefore, studies have been recently conducted to address these issues. For example, other parameters derived from VNIR bands, such as non-photosynthetically vegetation cover, solar albedo, and Normalized Difference Wetness Index (NDWI), were introduced to estimate LST for thermal sharpening [17-19]. In addition, more complex models, such as regression tree, artificial neural net, and physical models were applied in the sharpening procedure [5,20,21]. Many of the improvements rely on specific designing for certain land cover composite or landscape, which limit their wide applications. A recent effort by Gao [5] shows that regression-tree based technique has a potential to be adaptable for various data and landscapes. However, machine learning techniques might suffer from intensive computation cost and over-fitting risk [22]. Therefore, TsHARP remains the standard technique for comparison in the current researches $[23,24]$.

In this study, we attempted to combine another popular downscaling technique, spatial interpolation, to improve the accuracy and robustness of TsHARP. Various spatial interpolation techniques are widely used to enhance the spatial resolution of raster imagery. Among them, thin plate spline (TPS) [25,26] is considered as an effective and accurate method for simple parameter setting, good smoothness and robustness. Accordingly, TPS has been widely employed in spatial interpolation of geo-data, including meteorological data, DEM, etc. [27,28]. However, to our knowledge, there has been no attempt to use TPS in thermal sharpening. In this study, we combined the TPS and TsHAPR methods to enhance the advantages of each. The results of TPS and TsHARP are combined with the 
estimation of their errors in theory. In this manner, TPS result can be assigned with higher weights for the areas where TsHARP works poorly, and vice versa. Combining TPS and TsHARP is expected to improve both the accuracy and robustness of the thermal sharpening technique. The rest of paper is organized as follows. The details of the combined technique of TsHARP and TPS are introduced in Section 2; Section 3 describes a set of experiments for evaluating the proposed method across diverse landscapes; finally, Discussion and Conclusion are summarized in Sections 4 and 5.

\section{Methodology}

\subsection{Notations and Definitions}

For convenience and clarity, some symbols are defined here:

$n$ : the number of coarse pixels in low-resolution thermal imagery;

$m$ : the number of fine pixels covering the same area as a coarse pixel;

$N$ : the number of fine pixels in high-resolution VNIR imagery, which are equal to $n \times m$;

$(x, y)$ : row and column index of a certain pixel;

$i$ : index of a coarse pixel, $i=1, \ldots, n$;

$j$ : index of fine pixels in each coarse pixel, $j=1, \ldots, m$;

$T_{\text {high }}\left(x_{i j}, y_{i j}\right)$ : True LST at the fine pixel $\left(x_{i j}, y_{i j}\right)$;

$T_{\text {low }}\left(x_{i}, y_{i}\right)$ : LST at the coarse pixel $\left(x_{i}, y_{i}\right)$;

$\mathrm{NDVI}_{\text {high }}\left(x_{i j}, y_{i j}\right)$ : NDVI at the fine pixel $\left(x_{i j}, y_{i j}\right)$;

$\mathrm{NDVI}_{\text {low }}\left(x_{i}, y_{i}\right)$ : Aggregated NDVI at the coarse pixel $\left(x_{i}, y_{i}\right)$.

\subsection{TsHARP Method}

TsHARP assumes that there is a unique NDVI-LST relationship exists within a remotely sensed scene at multiple spatial resolutions. Firstly, an empirical relationship between NDVI and LST at the coarse thermal resolution is established.

$$
\begin{aligned}
T_{\text {low }}\left(x_{i}, y_{i}\right) & =f_{\text {reg }}\left[\operatorname{NDVI}_{\text {low }}\left(x_{i}, y_{i}\right)\right]+\varepsilon_{\text {reg }}\left(x_{i}, y_{i}\right) \\
& =a \cdot \operatorname{NDVI}_{\text {low }}\left(x_{i}, y_{i}\right)+b+\varepsilon_{\text {reg }}\left(x_{i}, y_{i}\right)
\end{aligned}
$$

where $a$ and $b$ are the regression coefficients estimated via the least-squares method, and $\varepsilon_{\text {reg }}\left(x_{i}, y_{i}\right)$ is the residual field at coarse pixel $\left(x_{i}, y_{i}\right)$. Only the simplest linear regression is considered because a suitable selection of LST proxy and regression tool is still problematic [11]. The regression equation is then applied to the fine pixels, and the LST at high spatial resolution is estimated as:

$$
\hat{T}_{\text {reg }}\left(x_{i j}, y_{i j}\right)=a \cdot \mathrm{NDVI}_{\text {high }}\left(x_{i j}, y_{i j}\right)+b
$$

Next, the final estimate of LST ( $\left.T_{\text {TsHARP }}\right)$ is derived by adding the coarse-resolution residual field back into the regression estimate:

$$
\begin{aligned}
T_{\text {TsHARP }}\left(x_{i j}, y_{i j}\right) & =\hat{T}_{\text {reg }}\left(x_{i j}, y_{i j}\right)+\varepsilon_{\text {reg }}\left(x_{i}, y_{i}\right) \\
& =\hat{T}_{\text {reg }}\left(x_{i j}, y_{i j}\right)+T_{\text {low }}\left(x_{i}, y_{i}\right)-\hat{T}_{\text {reg }}\left(x_{i}, y_{i}\right) \\
& =T_{\text {low }}\left(x_{i}, y_{i}\right)+a\left[\operatorname{NDVI}_{\text {low }}\left(x_{i}, y_{i}\right)-\operatorname{NDVI}_{\text {high }}\left(x_{i j}, y_{i j}\right)\right]
\end{aligned}
$$


TsHARP has a high accuracy in vegetation dominated areas; however, it might produce significant errors in low NDVI areas, such as urban areas, where LST could vary greatly as a result of the change of soil moisture and other factors.

\subsection{Thin Plate Spline (TPS) Interpolation}

TPS is a spatial interpolation technique for point data, which is also widely used for downscaling various geo-data. The basic principle of TPS is based on the spatial dependence of geo-data. Accordingly, it firstly fit a spatial dependent function that interpolates the known point data and minimizes the bending energy [24]. Then, data at any point on the surface can be interpolated by the fitted function. The basic TPS function is defined as:

$$
f_{\mathrm{TPS}}(x, y)=a_{0}+a_{1} x+a_{2} y+\frac{1}{2} \sum_{i=1}^{n} b_{i} r_{i}^{2} \log r_{i}^{2}
$$

with the following constrains:

$$
\sum_{i=1}^{n} b_{i}=\sum_{i=1}^{n} b_{i} x_{i}=\sum_{i=1}^{n} b_{i} y_{i}=0
$$

where $r_{i}^{2}=\left(x-x_{i}\right)^{2}+\left(y-y_{i}\right)^{2}$, and the coefficients are determined for satisfying:

$$
f_{\text {TPS }}\left(x_{i}, y_{i}\right)=T_{\text {low }}\left(x_{i}, y_{i}\right), \quad i=1, \ldots, n
$$

The TPS function can be fitted in a local spatial window or the entire image. In order to reduce the computation cost, TPS function in this study was fitted in the moving window of $5 \times 5$ coarse pixels. The TPS function was then used to acquire LST at high resolution without VNIR data input.

$$
\hat{T}_{\mathrm{TPS}}\left(x_{i j}, y_{i j}\right)=f_{\mathrm{TPS}}\left(x_{i j}, y_{i j}\right)
$$

Due to the fact that TPS interpolation considers only the spatial dependence of LST, it produces a result with a smooth spatial pattern in accordance with that coarse data exhibit. Compared to TsHARP, TPS cannot reconstruct the rich spatial details of LST image; however, it can produce a better result in the areas where NDVI poorly indicates LST and LST displays somewhat spatial continuity.

\subsection{Combination of TSHARP and TPS}

In order to enhance the advantages both of TsHARP and TPS, we developed a method which combines TsHARP and TPS. First, two high-resolution LST images were acquired by the sharpening of the regression method (Equation (2), TsHARP without residual correction) and TPS, respectively. Secondly, the Error of Regression Method and TPS were estimated respectively for each coarse pixel. Third, the weighted results of the regression method and TPS were combined with the estimation of their errors. Finally, new residual fields were added back to the weighted results. The flowchart is shown in Figure 1 and detailed procedures are given below. 
Figure 1. Flowchart of the proposed combined method of TsHARP and TPS.

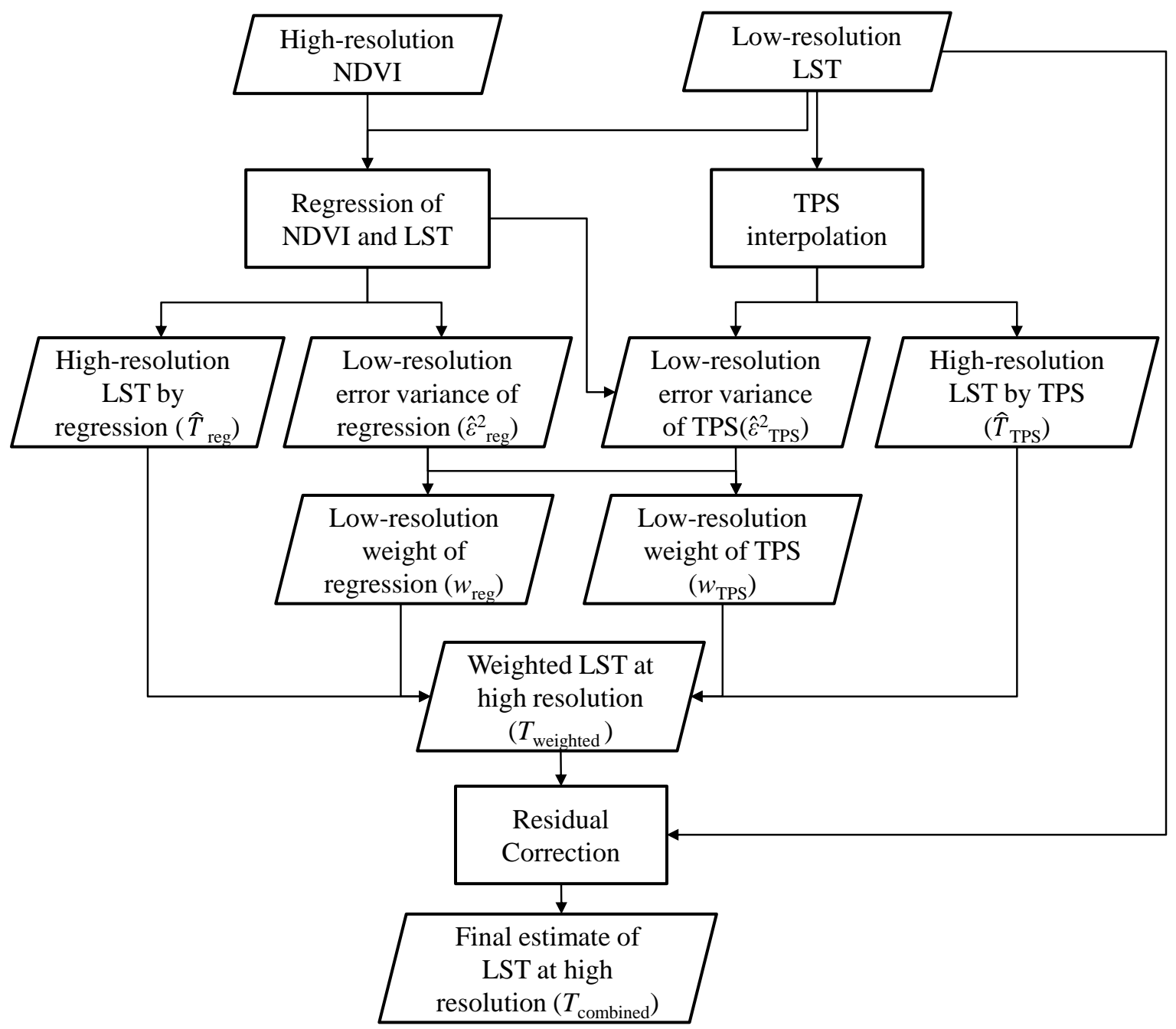

\subsubsection{Error Estimation of the Regression Method}

For the regression method, the error variance of regressed LST in coarse pixel $\left(x_{i}, y_{i}\right)$ can be calculated as the square of a coarse-resolution residual field if the error is assumed to be constant in a coarse pixel $\left(x_{i}, y_{i}\right)$

$$
\hat{\varepsilon}_{\text {reg }}^{2}\left(x_{i}, y_{i}\right)=\left[T_{\text {low }}\left(x_{i}, y_{i}\right)-\hat{T}_{\text {reg }}\left(x_{i}, y_{i}\right)\right]^{2}
$$

A larger coarse-resolution residual square in Equation (8) indicates a larger bias to the regression model for this coarse pixel, consequently it is a good estimate of error variance for the regression method.

\subsubsection{Error Estimation of TPS}

In general, TPS produces smoother LST imagery with a lack of spatial details because only spatial dependence is considered. Therefore, the spatial variance of downscaled LST by TPS is generally lower than that of true LST at same spatial scale. Assuming that total variance of true LST can be 
decomposed of the model variance and error variance, the error variance of sharpened LST in coarse pixel $\left(x_{i}, y_{i}\right)$ can be calculated as Equation (9):

$$
\hat{\varepsilon}_{\mathrm{TPS}}^{2}\left(x_{i}, y_{i}\right)=\left|\operatorname{Var}_{j}\left[T_{\text {high }}\left(x_{i j}, y_{i j}\right)\right]-\operatorname{Var}_{j}\left[\hat{T}_{\mathrm{TPS}}\left(x_{i j}, y_{i j}\right)\right]\right|
$$

where:

$$
\begin{aligned}
& \operatorname{Var}_{j}\left[T_{\text {high }}\left(x_{i j}, y_{i j}\right)\right]=\frac{1}{m} \sum_{j=1}^{m}\left[T_{\text {high }}\left(x_{i j}, y_{i j}\right)-T_{\text {low }}\left(x_{i}, y_{i}\right)\right]^{2} \\
& \operatorname{Var}_{j}\left[\hat{T}_{\text {TPS }}\left(x_{i j}, y_{i j}\right)\right]=\frac{1}{m} \sum_{j=1}^{m}\left[\hat{T}_{\text {TPS }}\left(x_{i j}, y_{i j}\right)-T_{\text {low }}\left(x_{i}, y_{i}\right)\right]^{2}
\end{aligned}
$$

Equations (10) and (11) are the total variance and model variance of LST in coarse pixel $\left(x_{i}, y_{i}\right)$ respectively. The absolute operation in Equation (9) is to address the abnormal case that total variance is less than model variance. As the true high-resolution $\operatorname{LST}\left(T_{\text {high }}\left(x_{i j}, y_{i j}\right)\right)$ is unknown, the total variance cannot be calculated directly. However, it can be estimated from the NDVI-LST regression model, as:

$$
\operatorname{Var}_{j}\left[T_{\text {high }}\left(x_{i j}, y_{i j}\right)\right]=b^{2} \times \underset{j}{\operatorname{Var}}\left[\operatorname{NDVI}\left(x_{i j}, y_{i j}\right)\right]+\operatorname{Var}\left(\varepsilon_{\text {reg }}\right)
$$

where:

$$
\operatorname{Var}_{j}\left[\operatorname{NDVI}\left(x_{i j}, y_{i j}\right)\right]=\frac{1}{m} \sum_{j=1}^{m}\left[\operatorname{NDVI}_{\text {high }}\left(x_{i j}, y_{i j}\right)-\operatorname{NDVI}_{\text {low }}\left(x_{i}, y_{i}\right)\right]^{2}
$$

and $\operatorname{Var}\left(\varepsilon_{\text {reg }}\right)$ is the residual variance in the NDVI-LST regression model at low resolution. Combined with Equation (9), the error variance of TPS at each coarse pixel is:

$$
\hat{\varepsilon}_{\mathrm{TPS}}^{2}\left(x_{i}, y_{i}\right)=\left|b^{2} \times \operatorname{Var}\left[\operatorname{NDVI}\left(x_{i j}, y_{i j}\right)\right]+\operatorname{Var}\left(\varepsilon_{\mathrm{reg}}\right)-\operatorname{Var}_{j}\left[\hat{T}_{\mathrm{TPS}}\left(x_{i j}, y_{i j}\right)\right]\right|
$$

Equation (14) means that the variance difference of the LST derived from NDVI-LST model and TPS can reflect the error of TPS. Such error estimation is empirical, however reasonable. In general, spatial variance of the LST sharpened by TPS is relatively small. If the NDVI variance in a coarse pixel is large, TPS cannot produce a good result because NDVI is the main factor determining LST. In contrast, if the NDVI variance is small, TPS produces better result as spatial dependence plays a more important role than NDVI in determining LST distribution. Especially, in the areas with low NDVI values, NDVI-LST relationship works poorly and spatial dependence should be considered more. Therefore, the proposed error estimation can reflect error of TPS to some extent.

\subsubsection{Combination of TPS and Regression Method}

Considering that Equations (8) and (14) are the estimates of the sharpening error variance of TPS and the regression method, the weights of these two methods are calculated for each coarse pixel:

$$
w_{\text {reg }}\left(x_{i}, y_{i}\right)=\frac{\hat{\varepsilon}_{\mathrm{TPS}}^{2}\left(x_{i}, y_{i}\right)}{\hat{\varepsilon}_{\mathrm{reg}}^{2}\left(x_{i}, y_{i}\right)+\hat{\varepsilon}_{\mathrm{TPS}}^{2}\left(x_{i}, y_{i}\right)}
$$




$$
w_{\mathrm{TPS}}\left(x_{i}, y_{i}\right)=\frac{\hat{\varepsilon}_{\mathrm{reg}}^{2}\left(x_{i}, y_{i}\right)}{\hat{\varepsilon}_{\mathrm{reg}}^{2}\left(x_{i}, y_{i}\right)+\hat{\varepsilon}_{\mathrm{TPS}}^{2}\left(x_{i}, y_{i}\right)}
$$

The sum of the two weight values is equal to one. Based on Bayesian principle, the weighted summation of the regression method and TPS is the best estimate [29]:

$$
T_{\text {weighted }}\left(x_{i j}, y_{i j}\right)=w_{\text {reg }}\left(x_{i}, y_{i}\right) \times \hat{T}_{\text {reg }}\left(x_{i j}, y_{i j}\right)+w_{\text {TPS }}\left(x_{i}, y_{i}\right) \times \hat{T}_{\text {TPS }}\left(x_{i j}, y_{i j}\right)
$$

Finally, a new coarse-resolution residual field is added back to the result:

$$
T_{\text {combined }}\left(x_{i j}, y_{i j}\right)=T_{\text {weighted }}\left(x_{i j}, y_{i j}\right)+T_{\text {low }}\left(x_{i}, y_{i}\right)-\frac{1}{m} \sum_{j=1}^{m} T_{\text {weighted }}\left(x_{i j}, y_{i j}\right)
$$

\section{Experiments}

\subsection{Simulation Experiment Using ASTER Data}

To investigate the effectiveness of the proposed combined method, we conducted a simulation experiment using subset images of ASTER level-1B (radiometric corrected VNIR bands) and level-2B03 (LST) products acquired of Yokohama city, Japan on 25 April 2004. Figure 2a shows the false color composite of this ASTER VNIR image, exhibiting very heterogeneous landscape. The low-resolution LST data was simulated by upscaling the LST image from the original 90-m resolution to 720 -m resolution (Figure $2 \mathrm{c}$ ). The upscaling was conducted by arithmetic averaging because other methods based on Stefan-Boltzmann law do not show obvious superiority [30]. Meanwhile, the NDVI image was also linearly resampled from $15-\mathrm{m}$ to $90-\mathrm{m}$ (Figure $2 \mathrm{~b}$ ). Then a simulation experiment of sharpening LST from 720-m to 90-m was conducted, and the original 90-m ASTER LST image served as the true high-resolution LST data for validation.

Figure 2. ASTER data in Yokohama city, Japan. (a) False color display of VNIR band; (b) 90-m NDVI image; (c) 720-m LST image.
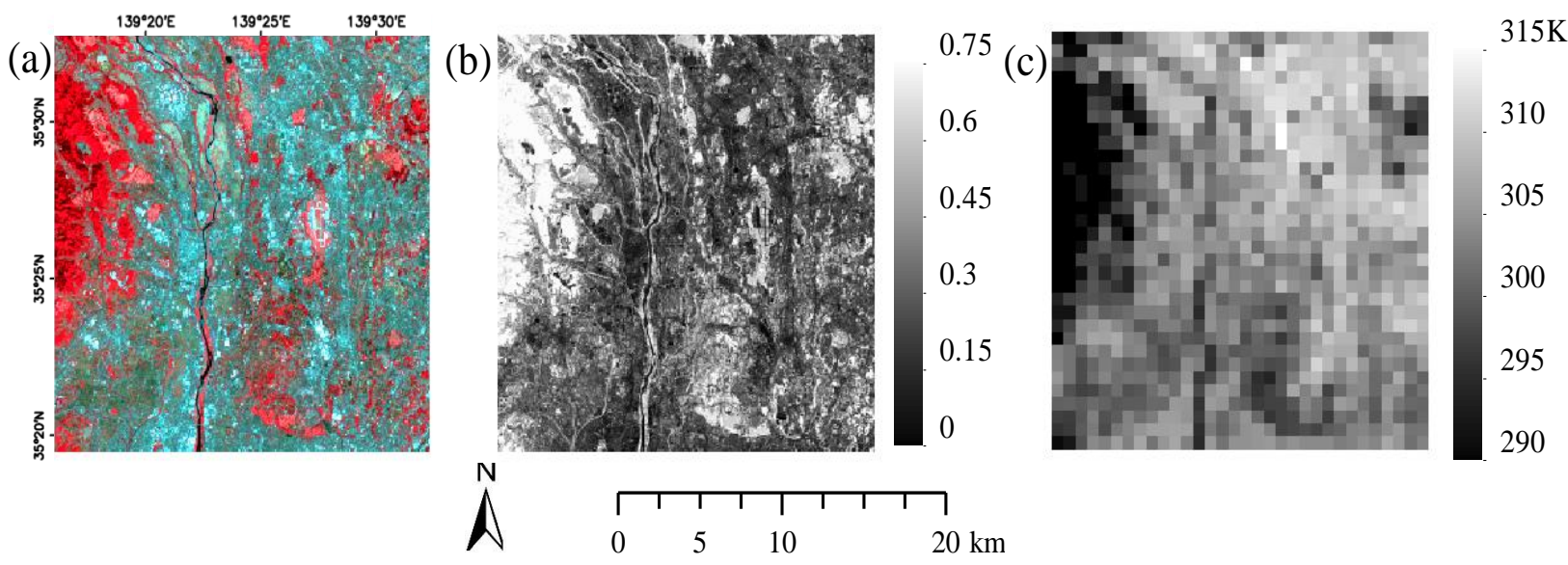

Figure 3 compares the true 90-m resolution LST image (Figure 3a) with the sharpened images by the combined method, TsHARP, and TPS (Figure 3b-d). TsHARP reconstructed rich spatial details of the LST image (Figure 3c), while TPS produced a much smoother spatial pattern (Figure 3d). In general, the result of TsHARP was more similar to the true LST image than that of TPS. However, 
TPS performed better than TsHARP in the areas where NDVI was unable to satisfactorily indicate LST. For example, TsHARP greatly overestimated the LST in the river area (Figure $3 \mathrm{~g}$ ) because water has a low NDVI and low LST, which is the reverse of the common NDVI-LST regression model. In contrast, TPS results were more accurate (Figure 3h). The proposed combined method produced a comprise result (Figure 3f). The sharpened error of TsHARP in the river area, although it was not completely corrected, was greatly reduced by utilizing the combined method. As shown in the scatter plots of estimated LST $v s$. true LST (Figure 3i-k), LST estimated by the proposed method was most similar to the true LST. Root mean square error (RMSE) of the proposed method $(2.24 \mathrm{~K})$ was lower than that of TsHARP $(2.48 \mathrm{~K})$ and TPS $(2.49 \mathrm{~K})$.

Figure 3. (a) True LST image at 90-m resolution; (b) Sharpened LST image by proposed method; (c) Sharpened LST image by TsHARP; (d) Sharpened LST image by TPS; (e) Enlarged image of true LST; (f) Enlarged image of LST sharpened by the proposed combined method; (g) Enlarged image of LST sharpened by TsHARP; (h) Enlarged image of LST sharpened by TPS; (i) Scatterplots of true LST and sharpened LST by proposed method; (j) Scatterplots of true LST and sharpened LST by TsHARP; (k) Scatterplots of true LST and sharpened LST by TPS.
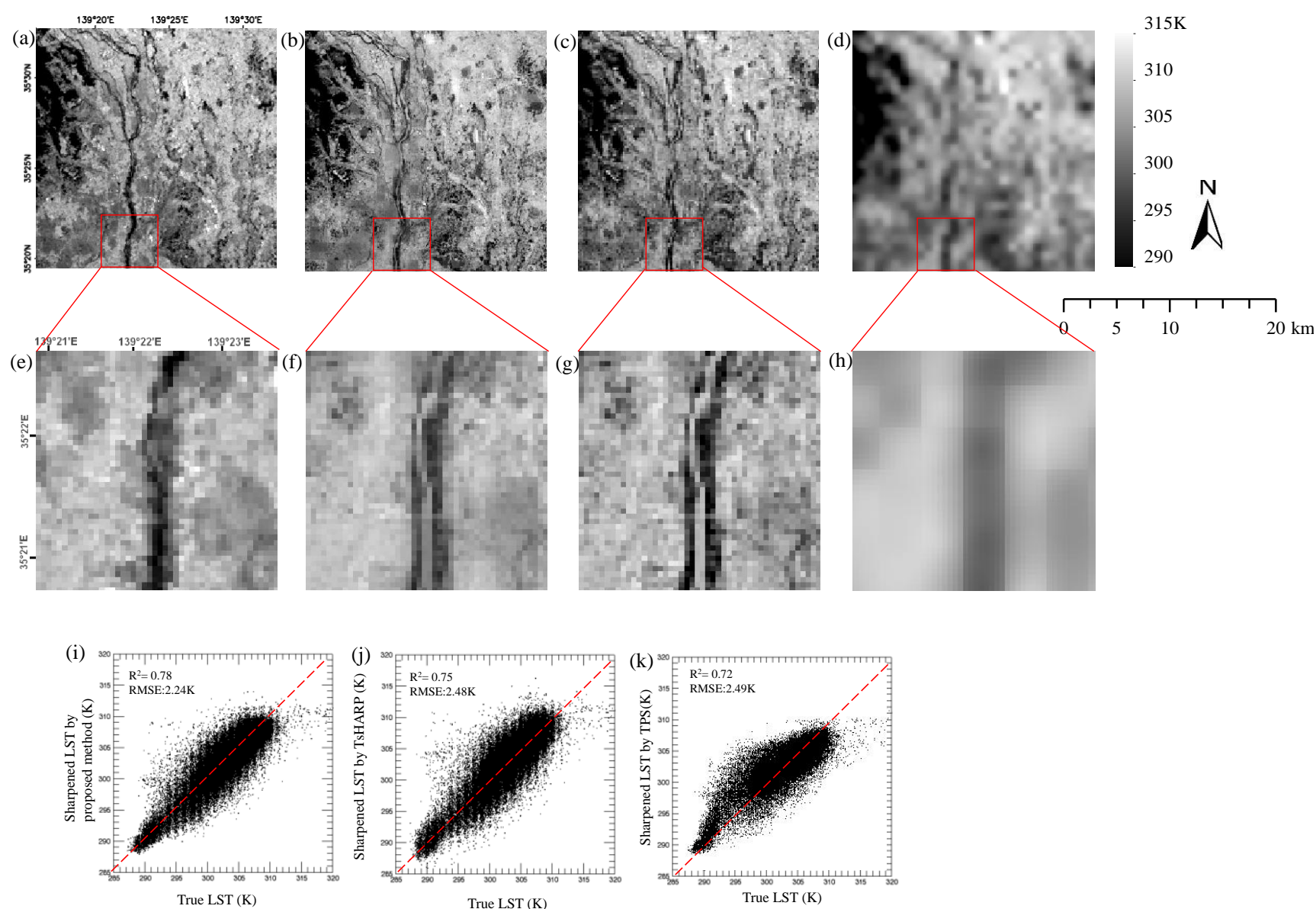

In order to further investigate when the combined method works well, the pixels were grouped into 10 sets according to their corresponding NDVI-LST regression residuals at low resolution. For each set, RMSEs of TsHARP and the proposed method were calculated. As shown in Figure 4, when 
the regression residual increased, both the RMSEs of the two methods also increased, while the RMSE of the proposed method increases in a slower manner. This indicates that the proposed method improves the sharpening accuracy of TsHARP for the areas where the residual of regression model is larger. This result further confirms that our proposed weights for the combination of the regression method and TPS were reasonable.

Figure 4. Relationship between Residual of NDVI-LST regression and sharpening RMSE by the proposed method and TsHARP.

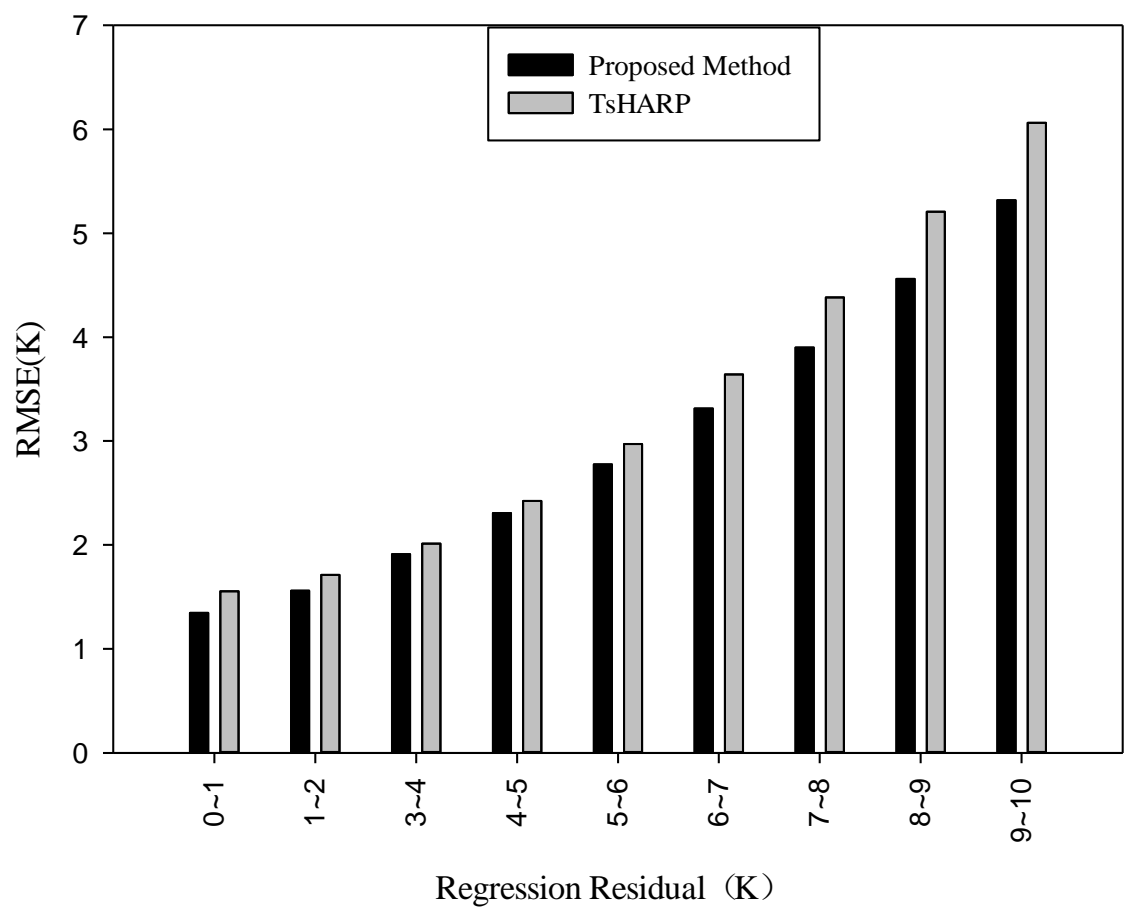

\subsection{Simulation Experiments across Different Landscapes}

To evaluate the robustness of the proposed method, more simulation experiments were conducted using different datasets. In addition to the ASTER imagery used in the Section 3.1, additional three satellite images acquired in different landscapes were used for the experiments. The information of the used satellite images for the new experiments is shown in Table 1 and Figure 5. For the Landsat ETM+ data, LST was calculated from thermal infrared band using the Mono-window algorithm [31]. The simulation sharpening experiments were conducted from different initial resolutions to target resolutions. For ASTER data, the resolution combinations (initial resolution to target resolution) included $1440 \mathrm{~m}$ to $90 \mathrm{~m}, 1440 \mathrm{~m}$ to $180 \mathrm{~m}, 1440 \mathrm{~m}$ to $360 \mathrm{~m}, 720 \mathrm{~m}$ to $90 \mathrm{~m}, 720 \mathrm{~m}$ to $180 \mathrm{~m}$, and $360 \mathrm{~m}$ to $90 \mathrm{~m}$. For Landsat ETM+ data, the resolution combinations included $960 \mathrm{~m}$ to $60 \mathrm{~m}$, $960 \mathrm{~m}$ to $120 \mathrm{~m}, 960 \mathrm{~m}$ to $240 \mathrm{~m}, 480 \mathrm{~m}$ to $60 \mathrm{~m}, 480 \mathrm{~m}$ to $120 \mathrm{~m}$, and $240 \mathrm{~m}$ to $90 \mathrm{~m}$. 
Table 1. Information of the satellite data for experiments.

\begin{tabular}{|c|c|c|c|c|c|}
\hline No. & $\begin{array}{l}\text { Data } \\
\text { Type }\end{array}$ & $\begin{array}{l}\text { Acquisition } \\
\text { Time }\end{array}$ & Location & $\begin{array}{c}\text { Landscape } \\
\text { (Dominant Percentage) }\end{array}$ & $\begin{array}{c}\text { Size (Thermal } \\
\text { Resolution) }\end{array}$ \\
\hline $\mathrm{a}$ & ASTER & 16 July 2010 & $\begin{array}{l}\text { Inner Mongolia, China } \\
\quad(44.6 \mathrm{~N}, 116.0 \mathrm{E})\end{array}$ & Grassland (90\%) & $\begin{array}{c}256 \times 256 \\
(90 \mathrm{~m})\end{array}$ \\
\hline $\mathrm{b}$ & ASTER & 26 April 2002 & $\begin{array}{c}\text { Haihe river basin, China } \\
\quad(38.3 \mathrm{~N}, 114.7 \mathrm{E})\end{array}$ & Cropland (70\%) & $\begin{array}{l}256 \times 256 \\
(90 \mathrm{~m})\end{array}$ \\
\hline $\mathrm{c}$ & ETM+ & 4 July 2001 & $\begin{array}{c}\text { Aichi prefecture, Japan } \\
\quad(35.2 \mathrm{~N}, 136.8 \mathrm{E})\end{array}$ & Rural (70\%) & $\begin{array}{l}256 \times 256 \\
(60 \mathrm{~m})\end{array}$ \\
\hline $\mathrm{d}$ & ASTER & 25 April 2004 & $\begin{array}{l}\text { Yokohama city, Japan } \\
\quad(35.4 \mathrm{~N}, 139.4 \mathrm{E})\end{array}$ & Urban (60\%) & $\begin{array}{l}256 \times 256 \\
(90 \mathrm{~m})\end{array}$ \\
\hline
\end{tabular}

Figure 5. Images and locations of the study areas. (a) Grassland in Inner Mongolia; (b) Cropland in Haihe river basin; (c) Rural area in Aichi prefecture; (d) Urban area in Yokohama city.

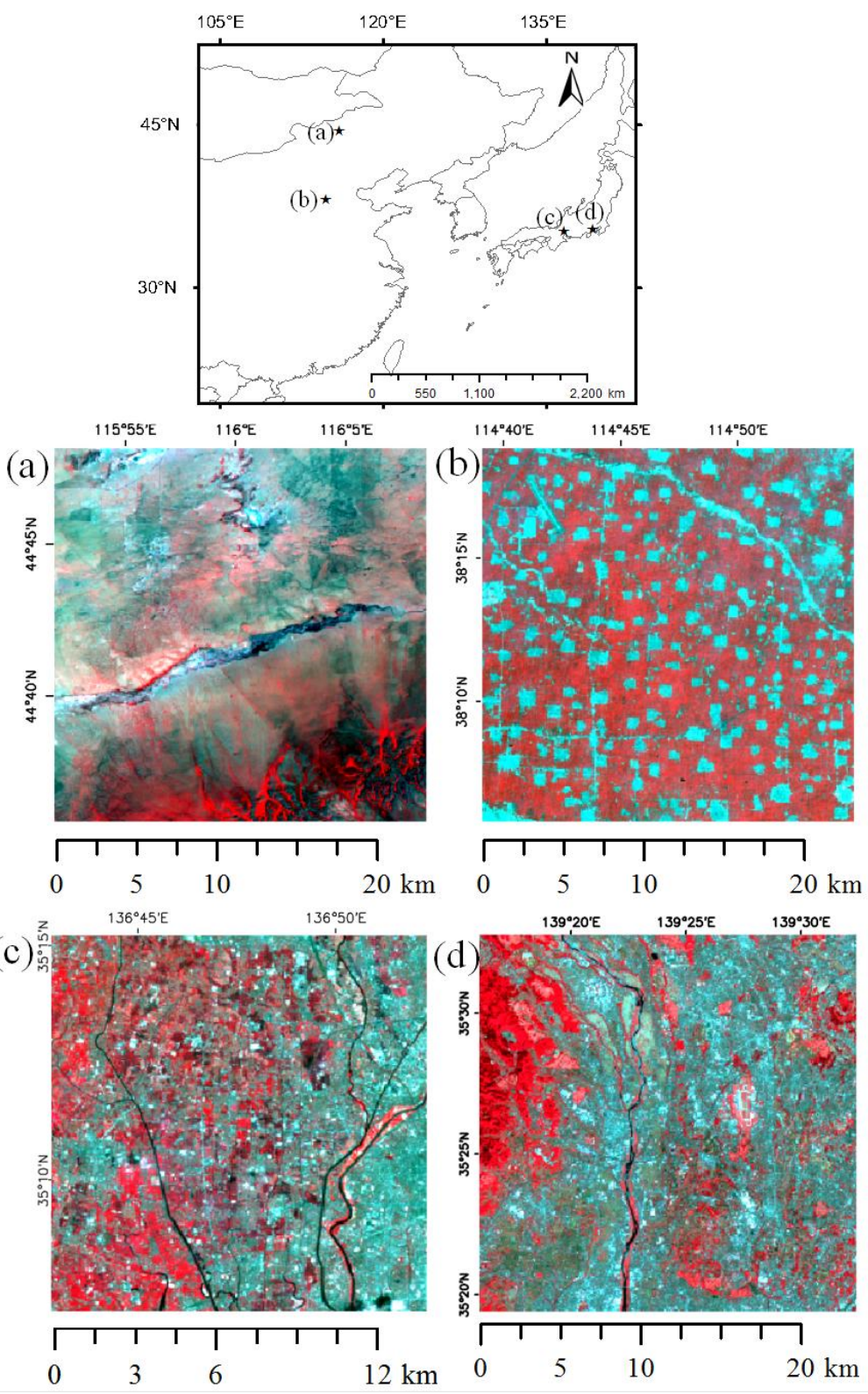


Figure 6. Thermal sharpening experiments (360-m to 90-m for ASTER and 240-m to 60-m for ETM+) in (a) grassland in Inner Mongolia; (b) cropland in Haihe river basin; (c) rural area of Aichi prefecture; and (d) urban area in Yokohama city.

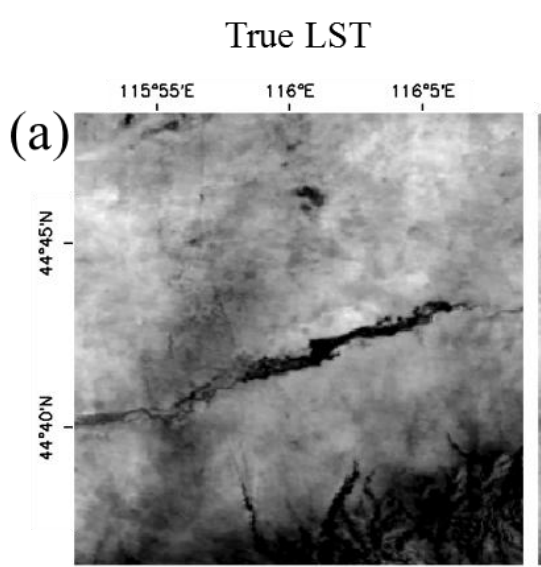

Proposed Method

TsHARP

Error of Proposed Method

Error of TsHARP
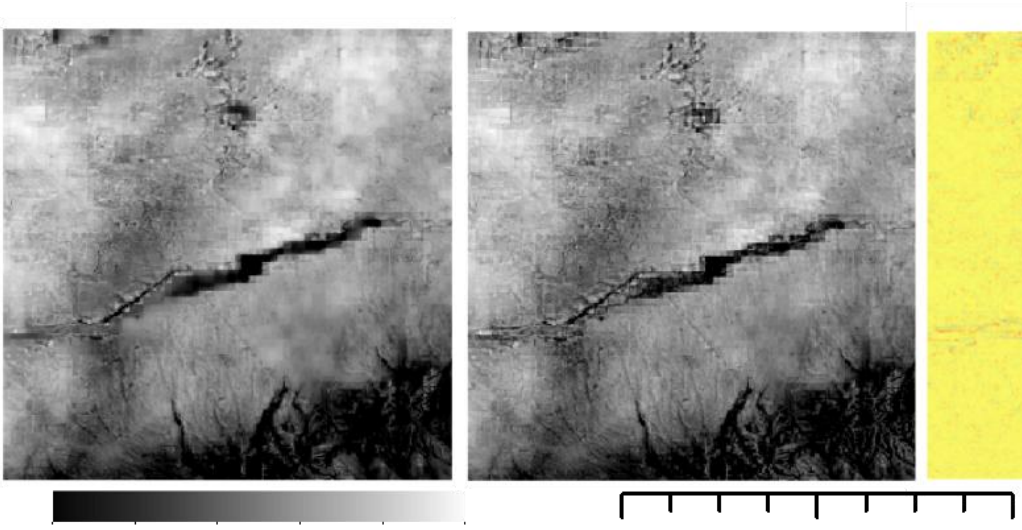

$\begin{array}{llllll}305 & 310 & 315 & 320 & 325 & 330 \mathrm{~K}\end{array}$

05

10

$20 \mathrm{~km}$
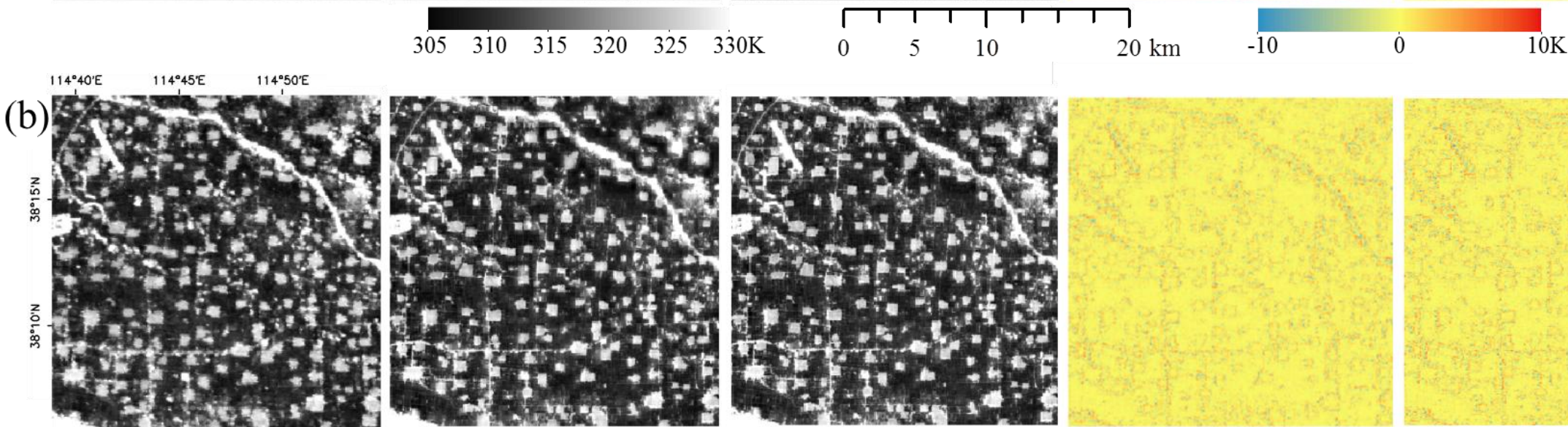

$290 \quad 295 \quad 300 \quad 305 \quad 310 \mathrm{~K}$
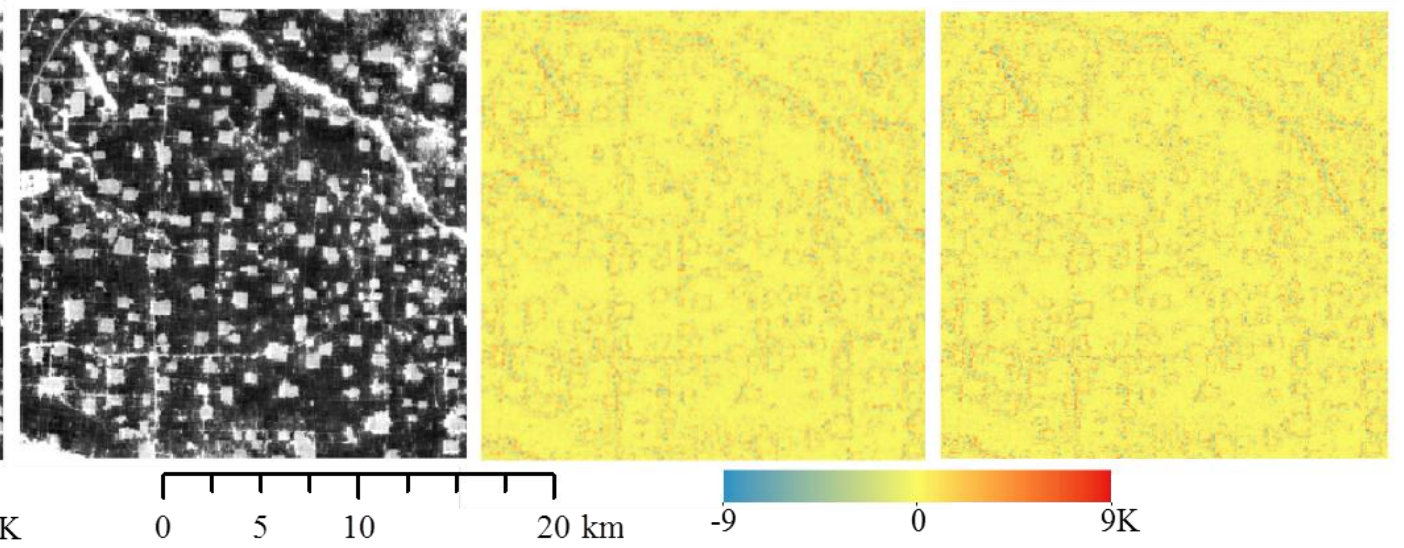
Figure 6. Cont.
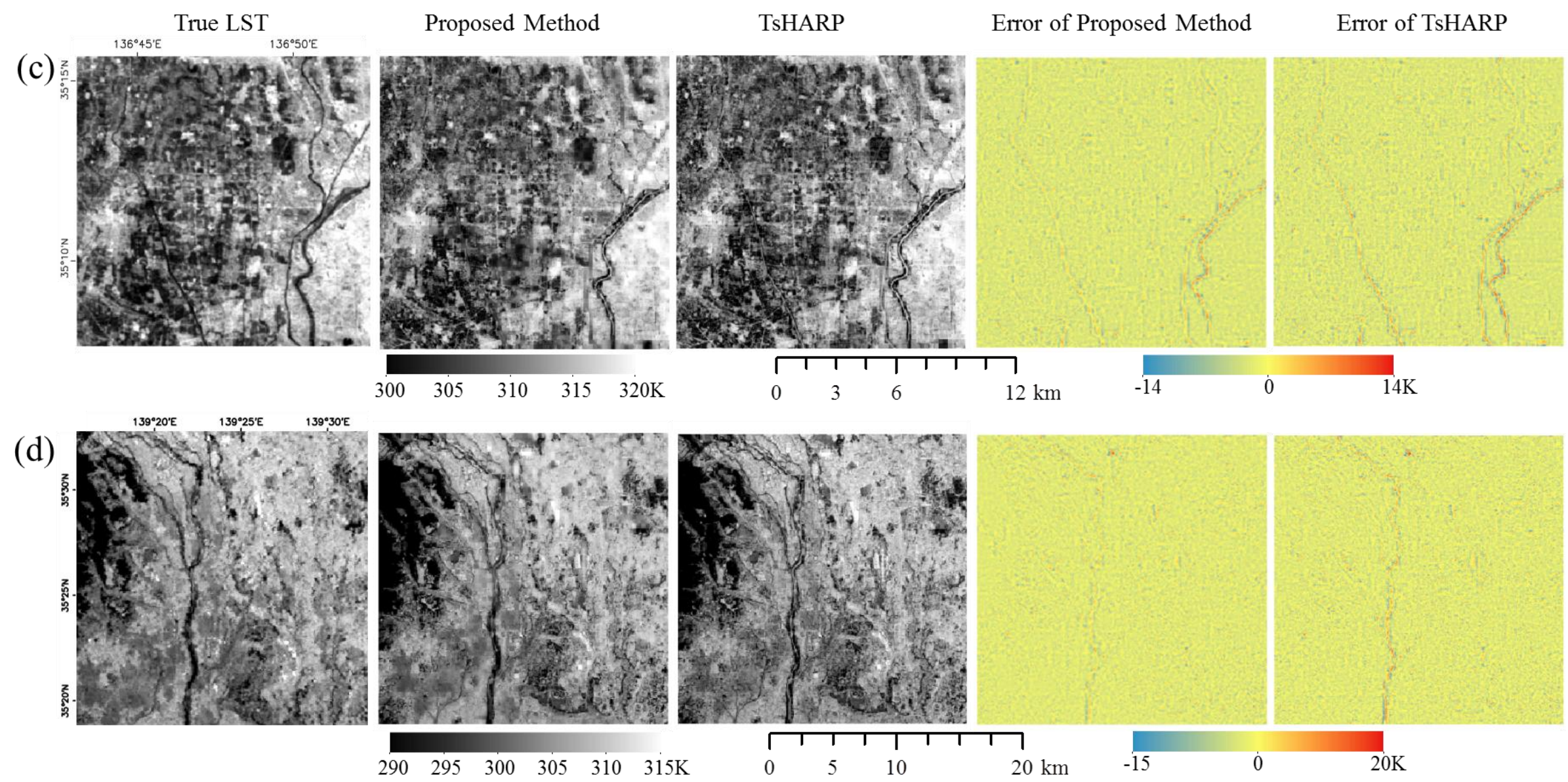
Figure 6 shows some examples of the sharpening results (360-m to 90-m for ASTER and 240-m to $60-\mathrm{m}$ for ETM+) by TsHARP and the combined method. Through the images of the sharpening error, it is found that the combined method produced smaller error than TsHARP especially for the water area. The river traces in the error images were largely reduced by the combined method. Significant differences are also found in the quantitative accuracy assessment. As shown in Figure 7, the proposed combined method showed lower RMSE than TsHARP for various landscapes and resolution-combinations with the exception of cropland landscape. For the cropland, the accuracy of the combined method was sometimes slightly lower than TsHARP. As the TsHARP method is particularly suitable for cropland [12,13], it acquires much higher accuracy for cropland than for other landscapes. Therefore, the potential of improvement by the combined method is very limited for this case. In summary, these experiments across various landscapes and resolution-combinations showed that the proposed method performed more accurately and robustly than TsHARP.

Figure 7. RMSEs of the sharpening experiments of proposed method and TsHARP for different landscapes and resolution-combinations. (a) Grassland in Inner Mongolia; (b) Cropland in Haihe river basin; (c) Rural area in Aichi prefecture; (d) Urban area in Yokohama city.

(a)
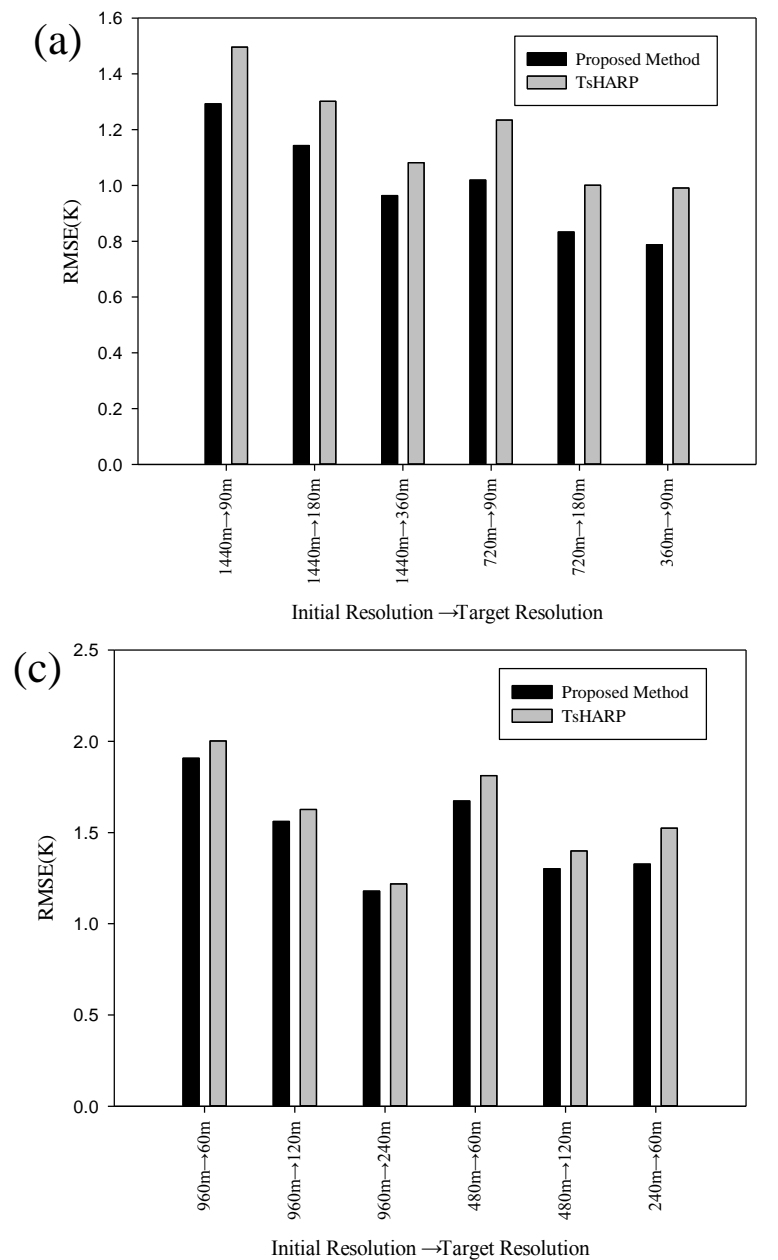

(b)

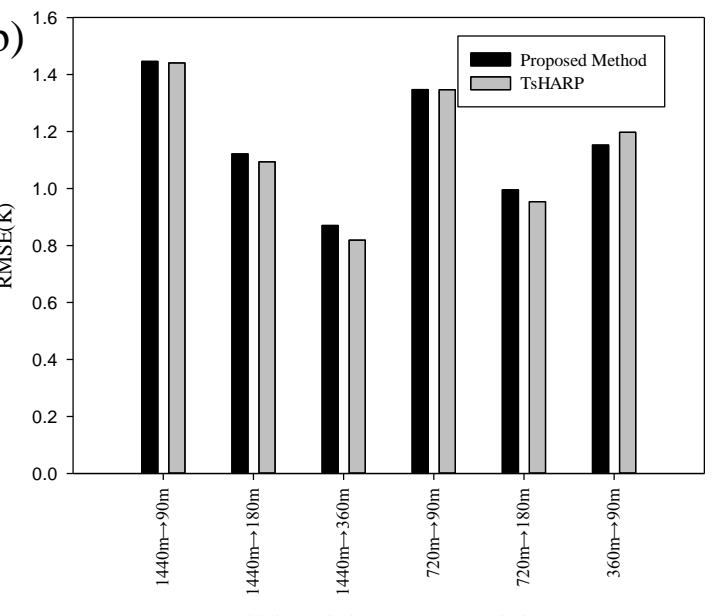

(d)

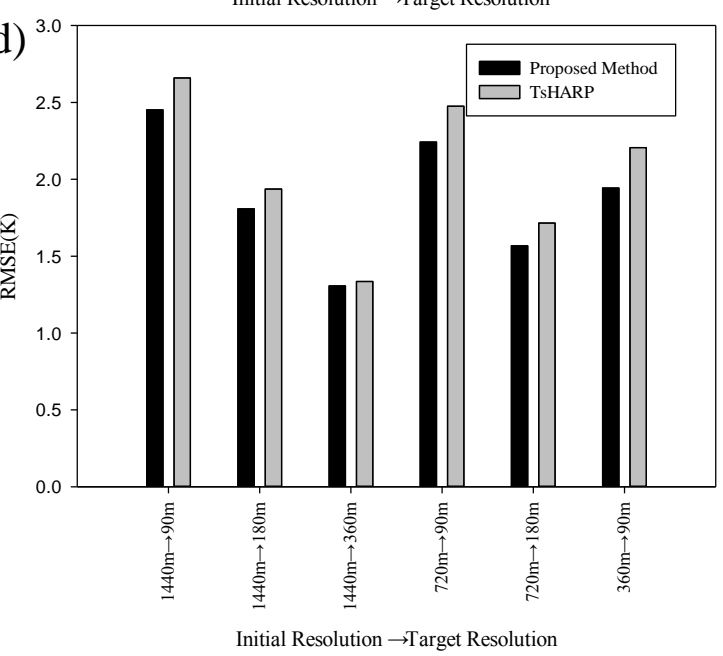


Figure 8. (a) 250-m MODIS NDVI image; (b) 1000-m MODIS LST image; (c) Sharpened 250-m LST image by proposed method; (d) Sharpened 250-m LST image by TsHARP; (e) Reprojected subset image from (c); (f) Reprojected subset image from (d); (g) True LST image resampled from ASTER data; (h) Scatterplots of true LST and sharpened LST by proposed method; (i) Scatterplots of true LST and sharpened LST by TsHARP.

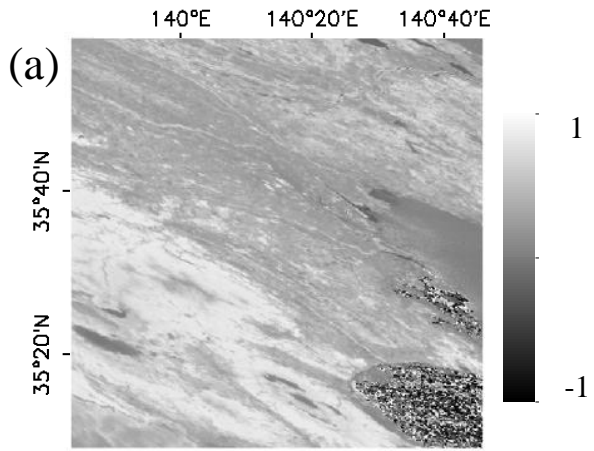

(b)

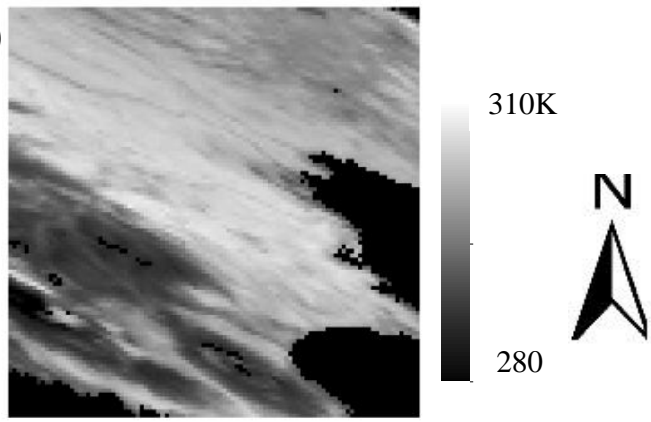

(c)

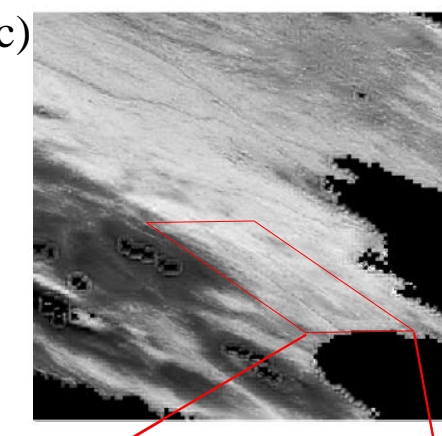

(d)
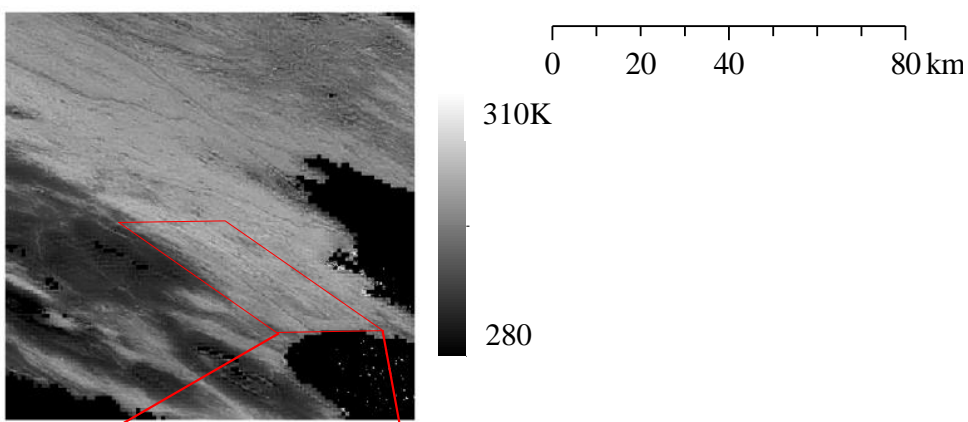

$310 \mathrm{~K}$

(e)
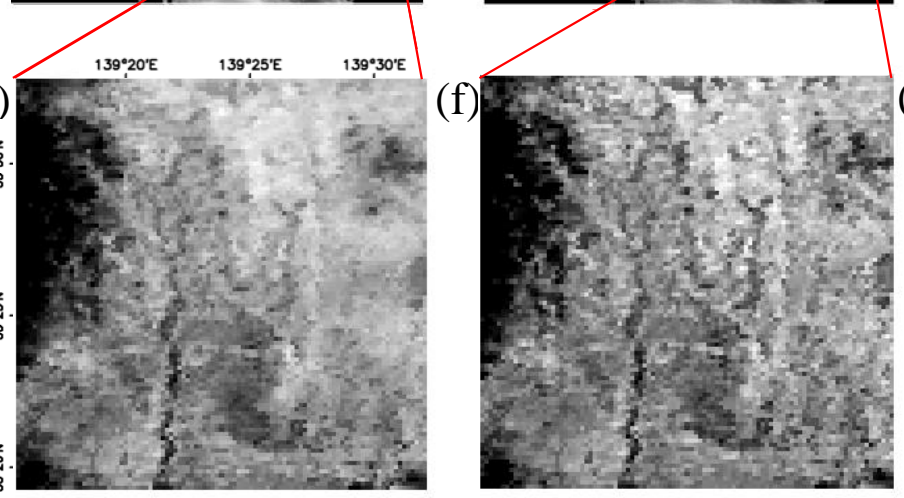

(g)

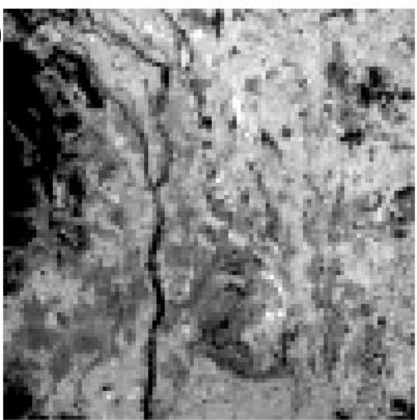

$315 \mathrm{~K}$

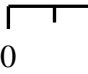

(h)

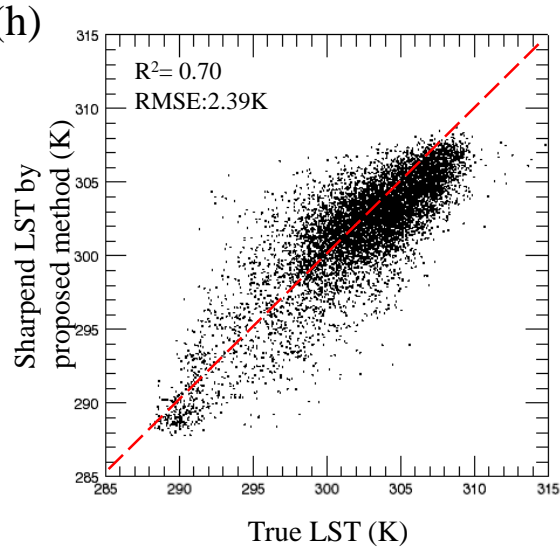

(i)

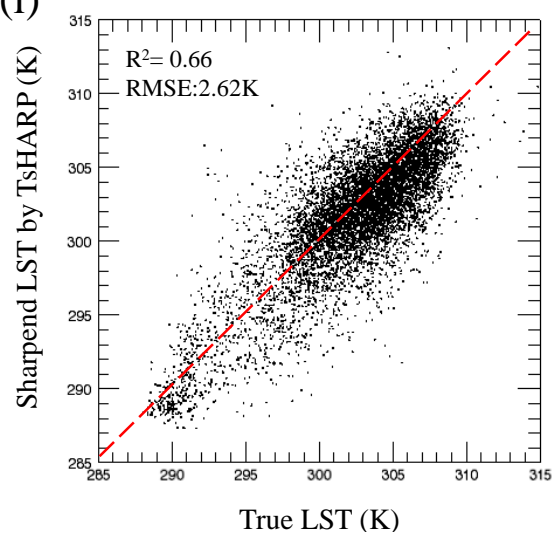




\subsection{Application of MODIS Data}

In addition to the simulation study, an application experiment using MODIS data was also conducted to evaluate our method. 250-m MODIS NDVI and 1000-m LST images acquired in Yokohama city on 25 April 2004, (Figure 8a,b) were used for thermal image sharpening. Two sharpened $250-\mathrm{m}$ resolution LST images were acquired by the proposed method and TsHARP, respectively, shown in Figure 8c,d. Then, a 250-m resolution LST image (Figure 8g) linearly resampled from 90-m ASTER LST image was served as the true LST image. The validation was conducted on the reprojected images of the corresponding subset of sharpened MODIS LST images (Figure 8e,f).

As shown in the scatterplots of two sharpened LST versus true LST (Figure 8h,i), the proposed combined method produced more accurate results than TsHARP. The RMSE of the proposed method was $2.38 \mathrm{~K}$, while the RMSE of the TsHARP method was $2.61 \mathrm{~K}$. This experiment confirmed that the proposed method outperforms the TsHARP not only in simulation experiments but also in practical application.

\section{Discussion}

The effectiveness of TsHARP relies on the good correlation between NDVI (or vegetation cover) and LST. However, the NDVI-LST relationship does not always endure in all the situations. A triangular is often found in scatterplot of NDVI and LST. As shown in the schematic of Figure 9 (revised from Figure 2 in [15]), the residuals of NDVI-LST regression model increase when the NDVI decreases because the evaporation vary greatly based on the moisture levels in the areas with low NDVI. As an extreme example, water area with low NDVI exhibit low LST, corresponding to the wet edge in Figure 9. For this case, TsHARP could produce large sharpening errors. TPS interpolation is a widely used downscaling technique for geo-data. It requires no assumptions, and performs robustly under a wide variety of situations. When prior knowledge is unavailable (e.g., NDVI-LST relationship does not work), TPS can acquire a comprise result without large errors although the spatial details cannot be reconstructed accurately. Thus, TPS could be a good supplement of TsHARP in areas where NDVI poorly indicates LST. For this reason, we combined TPS and TsHARP based on their error estimation in theory. The method with the larger error estimation is assigned with smaller weight; therefore, TPS is assigned with high weights in the areas where TsHARP works poorly for avoiding large sharpening error. It shows that the combined method outperforms TsHARP in most experiments. The exception is cropland landscape where TsHARP's performance is slightly better because the LST spatial pattern correlates well with NDVI. Accordingly, the combined method can only achieve similar accuracy with TsHARP in cropland landscape. In summary, the proposed combined method performs more robustly than TsHARP across various landscapes.

The proposed error estimation method was derived using some approximations. For example, the small error for a model at coarse resolution might correspond to a large error at fine-resolution. Moreover, the error estimation of TPS was acquired with empirical manner. However, the improvement of the combined method could prove the reasonability of the proposed error estimation indirectly. More advanced statistical model is encouraged to be developed for estimating the 
sharpening error of TsHARP and TPS interpolation in the future, by which the thermal sharpening result could be further refined.

Figure 9. Schematic of triangular in feature space of NDVI and LST.

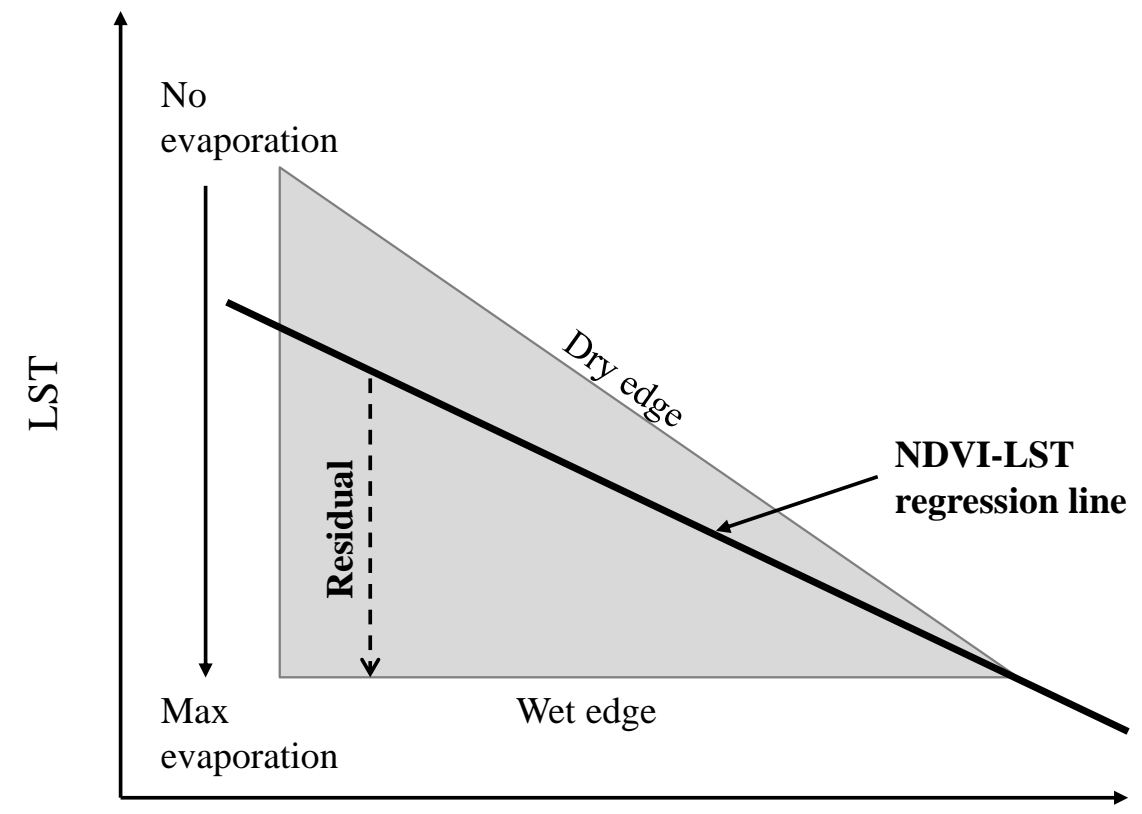

NDVI

Other than TPS, there are many spatial interpolation methods. Bilinear and cubic convolution interpolations are commonly used for resampling remote sensing data. Although the smoothness of the resampled images by these two methods is slightly weaker than that by TPS, they are also acceptable in most applications. Kriging is another popular interpolation technique that uses the variogram function. Although variogram analysis is helpful to understand the spatial dependence [32], the operation of Kriging interpolation is relatively complex because human intervention is required for the training of variogram model. It is expected that the sharpening accuracy could be further improved if new spatial interpolation technique specifically designed for thermal sharpening is developed.

\section{Conclusions}

In this study, a combined method of TsHARP and TPS (thin plate spline) interpolation was proposed for improving the accuracy and robustness of TsHARP for thermal sharpening. A set of sharpening experiments confirm the effectiveness of the proposed combined method based on error estimation. Without further parameter setting and assumptions, the proposed combined method is expected to be an effective thermal sharpening technique in the applications. Moreover, this study shows a potential of developing theoretical framework for combing different thermal sharpening methods. If the spatially explicit error of different methods can be reasonably estimated, the combined output is expected to be a better sharpening result. 


\section{Acknowledgments}

This work is supported by National Natural Science Foundation of China (41321001).

\section{Author Contributions}

Xuehong Chen wrote the manuscript and was responsible for the research design and algorithm development. Wentao Li coded the algorithm and made figures. Jin Chen and Yuhan Rao supported the algorithm design and interpretation of the results. Yasushi Yamaguchi provided the data and gave relevant technical support.

\section{Conflicts of Interest}

The authors declare no conflict of interest.

\section{References}

1. Gillespie, A.; Rokugawa, S.; Matsunaga, T.; Cothern, J.S.; Hook, S.; Khale, A.B. Temperature and emissivity separation algorithm for advanced spaceborne thermal emission and reflection radiometer (ASTER) images. IEEE Trans. Geosci. Remote Sens. 1998, 36, 1113-1126.

2. Kato, S.; Yamaguchi, Y. Analysis of urban heat-island effect using ASTER and ETM+ Data: Separation of anthropogenic heat discharge and natural heat radiation from sensible heat flux. Remote Sens. Environ. 2005, 99, 44-54.

3. Kustas, W.; Anderson, M. Advances in thermal infrared remote sensing for land surface modeling. Agric. For. Meteorol. 2009, 149, 2071-2081.

4. Weng, Q. Thermal infrared remote sensing for urban climate and environmental studies: Methods, applications, and trends. ISPRS J. Photogramm. Remote Sens. 2009, 64, 335-344.

5. Gao, F.; Kustas, W.P.; Anderson, M.C. A data mining approach for sharpening thermal satellite imagery over land. Remote Sens. 2012, 4, 3287-3319.

6. Cao, X.; Onishi, A.; Chen, J.; Imura, H. Quantifying the cool island intensity of urban parks using ASTER and IKONOS data. Landsc. Urban Plan. 2010, 96, 224-231.

7. Zhan, W.; Chen, Y.; Zhou, J.; Li, J.; Liu, W. Sharpening thermal imageries: A generalized theoretical framework from an assimilation perspective. IEEE Trans. Geosci. Remote Sens. 2011, 49, 773-789.

8. Chen, Y.; Zhan, W.; Quan, J.; Zhou, J.; Zhu, X.; Sun, H. Disaggregation of remotely sensed surface temperature: A generalized paradigm. IEEE Trans. Geosci. Remote Sens. 2014, in press.

9. Kustas, W.P.; Norman, J.M.; Anderson, M.C.; French, A.N. Estimating subpixel surface temperatures and energy fluxes from the vegetation index-radiometric temperature relationship. Remote Sens. Environ. 2003, 86, 429-440.

10. Agam, N.; Kustas, W.P.; Anderson, M.C.; Li, F.; Neale, C.M.U. A vegetation index based technique for spatial sharpening of thermal imagery. Remote Sens. Environ. 2007, 107, 545-558.

11. Zhan, W.; Chen, Y.; Zhou, J.; Wang, J.; Liu, W.; Voogt, J.; Zhu, X.; Quan, J.; Li, J. Disaggregation of remotely sensed land surface temperature: Literature survey, taxonomy, issues, and caveats. Remote Sens. Environ. 2013, 131, 119-139. 
12. Anderson, M.C.; Norman, J.M.; Mecikalski, J.R.; Torn, R.D.; Kustas, W.P.; Basara, J.B. A multiscale remote sensing model for disaggregating regional fluxes to micrometeorological scales. J. Hydrometeorol. 2004, 5, 343-363.

13. Agam, N.; Kustas, W.P.; Anderson, M.C.; Li, F.; Colaizzi, P.D. Utility of thermal sharpening over Texas high plains irrigated agricultural fields. J. Geophys. Res.: Atmos. 2007, 112, doi:10.1029/2007JD008407.

14. Chen, X.; Yamaguchi, Y.; Chen, J.; Shi, Y. Scale effect of vegetation-index-based spatial sharpening for thermal imagery: A simulation study by ASTER data. IEEE Geosci. Remote Sens. Lett. 2012, 9, 549-553.

15. Sandhlot, I.; Rasmussen, K.; Andersen, J. A simple interpretation of the surface temperature/vegetation index space for assessment of surface moisture status. Remote Sens. Environ. 2002, 79, 213-224.

16. Tang, R.; Li, Z.; Tang, B. An application of the Ts-VI triangle method with enhanced edges determination for evapotranspiration estimation from MODIS data in arid and semi-arid regions: Implementation and validation. Remote Sens. Environ. 2010, 114, 540-551.

17. Merlin, O.; Duchemin, B.; Hagolle, O.; Jacob, F.; Coudert, B.; Chehbouni, G.; Dedieu, G.; Garatuza, J.; Kerr, Y. Disaggregation of MODIS surface temperature over an agricultural area using a time series of Formosat-2 images. Remote Sens. Environ. 2010, 114, 2500-2512.

18. Dominguez, A.; Kleissl, J.; Luvall, J.C.; Rickman, D.L. High-resolution urban thermal sharpener (HUTS). Remote Sens. Environ. 2011, 115, 1772-1780.

19. Yang, G.; Pu, R.; Zhao, C.; Huang, W.; Wang, J. Estimation of subpixel land surface temperature using and endmember index based technique: A case examination on ASTER and MODIS temperature products over a heterogeneous area. Remote Sens. Environ. 2011, 115, 1202-1219.

20. Jing, L.H.; Cheng, Q.M. A technique based on non-linear transform and multivariate analysis to merge thermal infrared data and higher-resolution multispectral data. Int. J. Remote Sens. 2010, 31, 6459-6471.

21. Liu, D.; Zhu, X. An enhanced physical method for downscaling thermal infrared radiance. IEEE Geosci. Remote Sens. Lett. 2012, 9, 690-694.

22. Rogers, S.; Girolami, M. A First Course in Machine Learning; Chapman \& Hall/CRC, Taylor and Francis: Boca Raton, FL, USA, 2011.

23. Jiang, Y.; Weng, Q. Estimating LST using a vegetation-cover-based thermal sharpening technique. IEEE Geosci. Remote Sens. Lett. 2013, 10, 1249-1252.

24. Bindhu, V.M.; Narasimhan, B.; Sudheer, K.P. Development and verification of a non-linear disaggregation method (NL-DisTrad) to downscale MODIS land surface temperature to the spatial scale of Landsat thermal data to estimate evapotranspiration. Remote Sens. Environ. 2013, $135,118-129$.

25. Dubrule, O. Comparing splines and kriging. Comput. Geosci. 1984, 10, 327-338.

26. Wahba, G. Spline Models for Observational Data; SIAM: Philadelphia, PA, USA, 1990.

27. Boer, E.P.J.; de Beurs, K.M.; Hartkamp, A.D. Kriging and thin plate splines for mapping climate variables. Int. J. Appl. Earth Obs. Geoinf. 2001, 3, 146-154.

28. Chen, C.; Li, Y. A robust method of thin plate spline and its application to DEM construction. Comput. Geosci. 2012, 48, 9-16. 
29. Wikle, C.K.; Berliner, L.M. A Bayesian tutorial for data assimilation. Phys. D 2007, 230, 1-16.

30. Liu, Y.; Hiyama, T.; Yamaguchi, Y. Scaling of land surface temperature using satellite data: A case examination on ASTER and MODIS products over a heterogeneous terrain area. Remote Sens. Environ. 2006, 105, 115-128.

31. Qin, Z.; Karnieli, A.; Berliner, P. A mono-window algorithm for retrieving land surface temperature from Landsat TM data and its application to the Israel-Egypt border region. Int. J. Remote Sens. 2001, 22, 3719-3746.

32. Essa, W.; van der Kwast, J.; Verbeiren, B.; Batelaan, O. Downscaling of thermal images over urban areas using the land surface temperature-impervious percentage relationship. Int. J. Appl. Earth Obs.Geoinf. 2013, 23, 95-108.

(C) 2014 by the authors; licensee MDPI, Basel, Switzerland. This article is an open access article distributed under the terms and conditions of the Creative Commons Attribution license (http://creativecommons.org/licenses/by/3.0/). 